\title{
Tumor suppressor microRNA-613 inhibits glioma cell proliferation, invasion and angiogenesis by targeting vascular endothelial growth factor $\mathrm{A}$
}

\author{
XIONGWU YU ${ }^{1}$ and WEIMIN WANG ${ }^{2}$ \\ ${ }^{1}$ Department of Pediatric Surgery, Maternal and Child Health-Care Hospital of Qujing, Qujing; ${ }^{2}$ Department of \\ Neurosurgery, The First Affiliated Hospital of Kunming Medical University, Kunming, Yunnan 650032, P.R. China
}

Received June 3, 2016; Accepted May 12, 2017

DOI: $10.3892 / \mathrm{mmr} .2017 .7422$

\begin{abstract}
MicroRNAs (miRNAs) are small non-coding RNAs which can serve as oncogenes or tumor suppressors in glioma. The present study aimed to investigate the expression of miR-613 in glioma. Reverse transcription-quantitative polymerase chain reaction (RT-qPCR) was used to detect miR-613 in glioma cells and tissues and the relationship between miR-613 and vascular endothelial growth factor (VEGF) A was assessed using a luciferase reporter assay. In addition, glioma cells were transfected with miR-613 mimics and the mRNA and protein expression of VEGFA was detected using RT-qPCR and western blot analysis, respectively. The proliferative, invasive and tube formation capabilities of transfected cells were also assessed in vitro. Furthermore, a nude mouse tumor xenograft model was used to investigate the effects of miR-613 on tumor growth in vivo. The results of the present study demonstrated that the expression of miR-613 was decreased in glioma cell lines, and was associated with the grade of glioma. Ectopic expression of miR-613 markedly suppressed glioma cell proliferation and angiogenesis. Furthermore, the upregulation of miR-613 inhibited tumor angiogenesis and tumor growth in xenografted nude mice in vivo. VEGFA was demonstrated as a direct target of miR-613, as detected by western blot and luciferase reporter assays, and mediated miR-613 induced glioma cell proliferation and angiogenesis inhibition.
\end{abstract}

\section{Introduction}

Glioma, which is the most commonly occurring highly malignant primary brain tumor, comprises approximately one-third

Correspondence to: Dr Weimin Wang, Department of Neurosurgery, The First Affiliated Hospital of Kunming Medical University, 295 Xichang Road, Wuhua, Kunming, Yunnan 650032, P.R. China

E-mail: wangweimin2016@sina.com

Key words: microRNA-613, glioma, vascular endothelial growth factor A, proliferation, angiogenesis of intrinsic tumors of the central nervous system in both children and adults $(1,2)$. Although major advancements have been made by integrating neurosurgery, radiotherapy and chemotherapy to control malignant gliomas, poor prognosis and survival rate remains characteristic of such tumors $(3,4)$. Therefore, it is important to further understand the molecular mechanisms in glioma progression, and develop novel therapeutics.

MicroRNAs (miRNAs/miRs) are 21-23 nucleotide, small non-protein coding single-stranded RNA molecules, which are the important post-transcriptional regulators of gene expression in animals and plants (5). In animals, the regulatory effects of miRNAs are achieved by binding the complimentary sequences within the 3'-untranslated regions (UTRs) of their cognate messenger RNA (mRNA) targets $(6,7)$. miRNAs are involved in a variety of tumorigenic activities, including angiogenesis, proliferation, invasion and metastasis (8). Previous studies have suggested that the expression levels of many miRNAs are involved in glioma $(9,10)$. miR-613 has been identified to serve a key role in tumorigenesis and cancer progression, such as inhibition of ovarian cancer proliferation and invasion (11) and induction of lung cancer cell cycle arrest (12). However, the effect of miR-613 in glioma remains unknown.

The present study demonstrated that the mRNA expression level of miR-613 was decreased in glioma cell lines, and overexpression of miR-613 in both U87 and U251 cells suppressed invasion, proliferation and angiogenesis. In addition, vascular endothelial growth factor A (VEGFA) was identified to be a target gene of miR-613. The effects of miR-613 on tumor angiogenesis and proliferation were dependent on downregulation of VEGFA. These findings demonstrated the underlying mechanism and a novel role for miR-613 as a tumor inhibitor in glioma.

\section{Materials and methods}

Cell lines and patient samples. The NHA, H4, U87, U251 and SWO-38 human glioma cell lines and the HUVEC human endothelial cell line were purchased from the Institute of Biochemistry and Cell Biology of the Chinese Academy of Sciences (Shanghai, China). All cells were cultured in RPMI-1640 medium supplemented with penicillin and streptomycin (100 U/ml and $100 \mathrm{mg} / \mathrm{ml}$, respectively) and $10 \%$ fetal 
bovine serum (FBS; Gibco; Thermo Fisher Scientific, Inc., Waltham, MA, USA) at $37^{\circ} \mathrm{C}$ in a humidified atmosphere of $5 \% \mathrm{CO}_{2}$.

In the present study, 29 tissue samples were isolated from patients with stage I-II glioma and 24 samples from patients with stage III-IV glioma. In addition, 29 samples from normal adjacent tissue were also collected. The tissues were collected in the First Affiliated Hospital of Kunming Medical University (Kunming, China) between May 2014 and March 2016. Informed consent was obtained from each patient. All tissue samples had an affirmative pathological diagnosis and were classified on the basis of the World Health Organization criteria. None of the patients had received preoperative treatment, including chemotherapy or radiotherapy. A total of 32 male and 21 female patients were enrolled in the present study and the mean age of the patients was 69 years.

Lentivirus $(L V)$ production and transfection. The LV-miR-613 mimics and mimic controls were purchased from Shanghai GeneChem Co., Ltd. (Shanghai, China). Cells were plated in 6 -well plates at a density of $1 \times 10^{5}$ cells/well and were transfected with $200 \mu \mathrm{l}(100 \mathrm{nM}) \mathrm{miR}-613$ mimics or mimic controls using Lipofectamine 2000 reagent (Invitrogen; Thermo Fisher Scientific, Inc.) according to the manufacturer's protocol. The transfected cells were cultured in RPMI-1640 medium supplemented with $10 \% \mathrm{FBS}$ for $48 \mathrm{~h}$ at $37^{\circ} \mathrm{C}$ in a humidified atmosphere of $5 \% \mathrm{CO}_{2}$. The sequences of the oligonucleotides that were used were as follows: miR-613 mimic sense, 5'-AGG AAU GUU CCU UCU UUG CC-3' and antisense, 5'-UGG CAA AGA AGG AAC AUU CCU UG-3'; negative control (NC) sequence sense, 5'-ACU ACU GAG UGA CAG UAG A-3' and antisense, 5'-AUC UAC UGU CAC UCA GUA GUG G-3'.

Plasmid construction and dual luciferase activity assay. TargetScan (http://targetscan.org/) and MiRanda (http:// www.microrna.org/) software were used to predict the potential target genes of miR-613, and VEGFA was identified as a possible target. The eukaryotic expression vector pcDNA3.1 (+) was subcloned with full-length VEGFA cDNA (Invitrogen; Thermo Fisher Scientific, Inc.). The VEGFA 3'-UTR target site for miR-613 was amplified by polymerase chain reaction (PCR) and cloned into the XbaI site of a pGL3 control vector (Promega Corp., Madison, WI, USA). This vector was termed wild-type (WT) VEGFA 3'-UTR. The Quick-Change Mutagenesis kit (Agilent Technologies, Inc., Santa Clara, CA, USA) was used to carry out the site-directed mutagenesis of the miR-613 target-site in the VEGFA 3'UTR, termed mutant (Mut) VEGFA 3'-UTR. For the luciferase activity assay, U87 cells were seeded at density of $5 \times 10^{4}$ cells/well in 24-well plates and co-transfected with WT or Mut VEGFA 3'UTR vectors and the control vector pRL-(cytomegalovirus) CMV coding for Renilla luciferase, (Promega Corp.) using Lipofectamine 2000 reagent (Invitrogen; Thermo Fisher Scientific, Inc.) according to the manufacturer's protocol. A Dual-Luciferase Reporter Assay system (Promega Corp.) was used to detect the luciferase activity after transfection for $36 \mathrm{~h}$.

RNA extraction and reverse transcription-quantitative $(R T q)-P C R$. Total RNA was extracted with TRIzol reagent
(Invitrogen; Thermo Fisher Scientific, Inc.) according to the manufacturer's protocol. The mRNA expression level of miR-613 was detected using an ABI PRISM 7500 Sequence Detection system (ABI) using TaqMan MicroRNA assay kits (Applied Biosystems, Foster City, CA, USA) according to the manufacturer's protocol. The thermocycling conditions that were used were as follows: Initial denaturation at $95^{\circ} \mathrm{C}$ for $10 \mathrm{~min}$, followed by 40 cycles at $95^{\circ} \mathrm{C}$ for $15 \mathrm{sec}$ and at $60^{\circ} \mathrm{C}$ for $60 \mathrm{sec}$. U6 served as the control for normalization.

The gene expression of VEGFA was also detected. Total RNA was reverse transcribed into cDNA using Revert Aid First Strand cDNA Synthesis kit (Thermo Fisher Scientific Inc.) according to the manufacturer's protocol. The temperature conditions used were as follows: At $25^{\circ} \mathrm{C}$ for $5 \mathrm{~min}$, at $42^{\circ} \mathrm{C}$ for $60 \mathrm{~min}$ and at $70^{\circ} \mathrm{C}$ for $10 \mathrm{~min}$. qPCR was performed on cDNA using SYBR-Green (Takara Bio, Inc., Otsu, Japan) and gene expression was normalized to $\beta$-actin. Thermocycling conditions were as follows: At $95^{\circ} \mathrm{C}$ for $30 \mathrm{sec}$, followed by 40 cycles at $95^{\circ} \mathrm{C}$ for $5 \mathrm{sec}$ and at $60^{\circ} \mathrm{C}$ for $34 \mathrm{sec}$. The primers that were used in the present study were as follows: VEGFA forward, 5'-ATC CAA TCG AGA CCC TGG TG-3' and reverse, 5'-ATC TCT CCT ATG TGC TGG CC-3'; $\beta$-actin forward, 5'-TGA GAG GGA AAT CGT GCG TGA C-3' and reverse, 5'-GCT CGT TGC CAAT AGT GAT GAC C-3'; miR-613 forward, 5'-AGG AAT GTT CCT TCT-3' and reverse, 5'-GTG CAG GGT CCG AGG T-3'; and U6 forward, 5'-CTC GCT TCG GCA GCA CA-3' and reverse, 5'-AAC GCT TCA CGA ATT TGC GT-3'. The specificity of primer sequences were detected by its dissociation curve, and the $2^{-\Delta \Delta \mathrm{Cq}}$ (quantitation threshold) method was used to calculate the relative gene expression levels (13).

Western blot analysis. Western blot assay was conducted as previously described (14). Briefly, cells were lysed with ice-cold lysis buffer (Cell Signaling Technology, Inc., Danvers, MA, USA). The concentrations of proteins were measured using a bicinchoninic acid protein assay kit (Thermo Fisher Scientific, Inc.) according to the manufacturer's protocol. Total proteins $(30 \mu \mathrm{g})$ were separated by $10 \%$ SDS-PAGE, transferred onto polyvinylidene difluoride membranes (EMD Millipore, Billerica, MA, USA) and blocked in 5\% skim milk (BD Biosciences, Franklin Lakes, NJ, USA) for $2 \mathrm{~h}$ at room temperature. Membranes were then incubated with the following primary antibodies at $4^{\circ} \mathrm{C}$ overnight: Anti-VEGFA (cat no. ab1316; 1:1,000; Abcam, Cambridge, UK), anti-angiopoietin-2 (Ang-2; cat no. sc-20718; 1:50), anti-CD31 (cat. no. sc-71872; 1:200) (both from Santa Cruz Biotechnology, Inc., Dallas, TX, USA) and anti-GAPDH (cat no. ab9484; 1:5,000; Abcam). Subsequently, they were incubated with the following horseradish peroxidase (HRP)-conjugated secondary antibodies for $1 \mathrm{~h}$ at room temperature: Anti-mouse immunoglobulin (Ig) G (cat no. sc-2005; 1:2,000; Santa Cruz Biotechnology, Inc.) and anti-rabbit IgG (cat no. sc-2004; 1:2,000; Santa Cruz Biotechnology, Inc.). Enhanced chemiluminescence (GE Healthcare, Chicago, IL, USA) was used to detect the expression levels of the target proteins. Blots were semi-quantified using Image Lab software (Bio-Rad Laboratories, Inc., Hercules, CA, USA).

Immunohistochemistry. According to the manufacturer's protocol, immunohistochemistry for VEGFA and CD31 was 
performed on 4- $\mu \mathrm{m}$ formalin fixed, paraffin-embedded tissue sections. The sections were incubated with anti-VEGFA (1:200) and anti-CD31 (1:50) primary antibodies overnight at $4^{\circ} \mathrm{C}$. The sections were then incubated for $1 \mathrm{~h}$ at room temperature with HRP-conjugated anti-mouse secondary antibody (cat. no. sc-2005; 1:200; Santa Cruz Biotechnology, Inc.). Immunohistochemical stains were completed using an automated immunostainer (Ventana Medical Systems Inc., Tucson, AZ, USA) and stained sections were observed under a light microscope.

Cell proliferation and tube formation assay. For the colony formation test, U87 and U251 cells (1,000 cells/well) were treated with miRNA control, miR-613 mimc, or miR-613 mimic and pcDNA3.1-VEGFA, seeded into 6-well plates and cultured for 14 days. The colonies (>50 cells; diameter, 60-100 $\mu \mathrm{m}$ ) were stained with $0.1 \%$ methylene blue for $5 \mathrm{~min}$ at room temperature and imaged using an optical microscope. The tube formation assay was conducted as previously described (14), in 24-well plates using growth factor reduced Matrigel (BD Biosciences). Cells ( $2 \times 10^{4}$ cells/well) were resuspended in serum-free medium and plated on the Matrigel-coated plates. Following incubation at $37^{\circ} \mathrm{C}$ overnight, each well was observed under a light microscope. Tubules in each field were imaged and an average of tubules from 3-5 random fields of view/well was counted.

Cell invasion assay. The cell invasion assay was conducted as previously described (5) using Matrigel-coated Transwell inserts (Costar; Corning Incorporated, Corning, NY, USA). Briefly, $50 \mu 1(2.0 \mathrm{mg} / \mathrm{ml})$ Matrigel (BD Biosciences) was added in the upper chambers of the Transwell inserts for $30 \mathrm{~min}$ at $37^{\circ} \mathrm{C}$. Subsequently, $3 \times 10^{3}$ cells in RPMI- 1640 medium without serum were seeded in the upper chambers of the inserts. In the lower chambers, $600 \mu 1$ culture medium supplemented with $10 \%$ FBS was added as a chemoattractant. Following $24 \mathrm{~h}$ incubation, non-invaded cells were removed using a cotton swab. Cells that had invaded the lower membrane were fixed with $4 \%$ paraformaldehyde for $30 \mathrm{~min}$ at room temperature, stained with $0.1 \%$ crystal violet for $20 \mathrm{~min}$ at room temperature, and photographed under a phase-contrast microscope (Olympus Corp., Tokyo, Japan). A total of 5 random fields of view were selected for cell counting.

Animal tumor studies. Animal experiments were approved by the Ethics Committee of Health and Planning Commission of Qujing City (Qujing, China). A total of 20 male BALB/c nude mice (age, 5-6 weeks; weight, 18-22 g) were purchased from Shanghai SLAC Laboratory Animal Co., Ltd. (Shanghai, China). Mice were housed in a specific-pathogen-free animal center and fed with autoclaved water and laboratory rodent chow ad libitum. Mice were maintained in a controlled temperature $\left(21-23^{\circ} \mathrm{C}\right)$ under a 12 -h light/dark cycle, in standard vinyl cages with air filter tops. Cages, bedding and water containers were autoclaved prior to use. At the beginning of the experiment, mice were subcutaneously injected in the front right leg with $0.2 \mathrm{ml} \mathrm{U87}$ cells $\left(2.5 \times 10^{6}\right)$, which had been previously transfected with miRNA control or miR-613 mimics using Lipofectamine 2000 reagent (Invitrogen; Thermo Fisher Scientific, Inc.) according to the manufacturer's protocol. Tumor sizes were recorded at the indicated time points, and tumor volume $\left(\mathrm{mm}^{3}\right)$ was calculated as follows: Tumor volume $=1 / 2$ (tumor length) $\mathrm{x}$ (tumor width) ${ }^{2}$. On day 42 post-injection, mice were sacrificed and the tumor nodules were dissected and weighed.

Statistical analysis. All data are presented as the mean \pm standard deviation of 3 independent experiments. One-way analysis of variance or two-tailed Student's t-test were used to analyze the significant differences between groups. SPSS 18.0 (SPSS, Inc., Chicago, IL, USA) and GraphPad Prism 6.0 (National Institutes of Health, Bethesda, MD, USA) software were used for data analysis. $\mathrm{P}<0.05$ was considered to indicate a statistically significant difference.

\section{Results}

miR-613 expression is downregulated in glioblastoma multiforme (GBM) cell lines and is associated with the glioma grade. The expression level of miR-613 was detected in NHA, H4, U87, U251 and SWO-38 cell lines by RT-qPCR. The results demonstrated that the mRNA expression level of miR-613 was decreased in high-grade glioma cell lines (U87, U251 and SWO-38) compared with NHA cells and the low-grade glioma cell line H4 (P<0.05; Fig. 1A). As U87 and U251 cells had the lowest expression of miR-613, they were selected for subsequent experiments. To further confirm the different expression levels of miR-613 in different grade gliomas, the mRNA expression level of miR-613 in different grade glioma tissues was examined by RT-qPCR. As a result, miR-613 levels in glioma I-II were significantly reduced compared with normal adjacent tissues (NATS), and in glioma III-IV it was the lowest (Fig. 1B).

miR-613 serves as a regulator of VEGFA by attaching to VEGFA 3'-UTR. MiRanda algorithms and TargetScan were used to search for target genes of miR-613. Among the mRNAs involving miR-613 recognition sites in their 3'-UTRs, VEGFA was focused on. In order to confirm that VEGFA was a direct target of miR-613, VEGFA wild-type (WT) or mutant 3'-UTR was subcloned into a luciferase reporter vector and co-transfected with miR-613 mimics or a negative control into U87 and U251 cells (Fig. 2A). The results demonstrated that in U87 and U251 cell lines, miR-613 significantly inhibited the luciferase activity of the VEGFA WT 3'-UTR, but had no influence on the mutant (Fig. 2B and C). To evaluate the effect of miR-613 on VEGFA expression, U87 and U251 cells were transfected with miR-613 mimics and control. The results demonstrated that overexpression of miR-613 downregulated the mRNA and protein expression levels of VEGFA (Fig. 2D and E). These findings illustrated that VEGFA was a direct downstream target of miR-613 in GBM cells.

miR-613 suppresses the proliferation, invasion and angiogenesis of GBM cells via targeting VEGFA in vitro. VEGFA has been identified to be potentially the most important mediator in glioma. Previous studies demonstrated that VEGFA serves a key role in regulating tumor-induced angiogenesis in glioma $(15,16)$. Studies have indicated that overexpression of VEGFA was associated with poor prognosis and the 

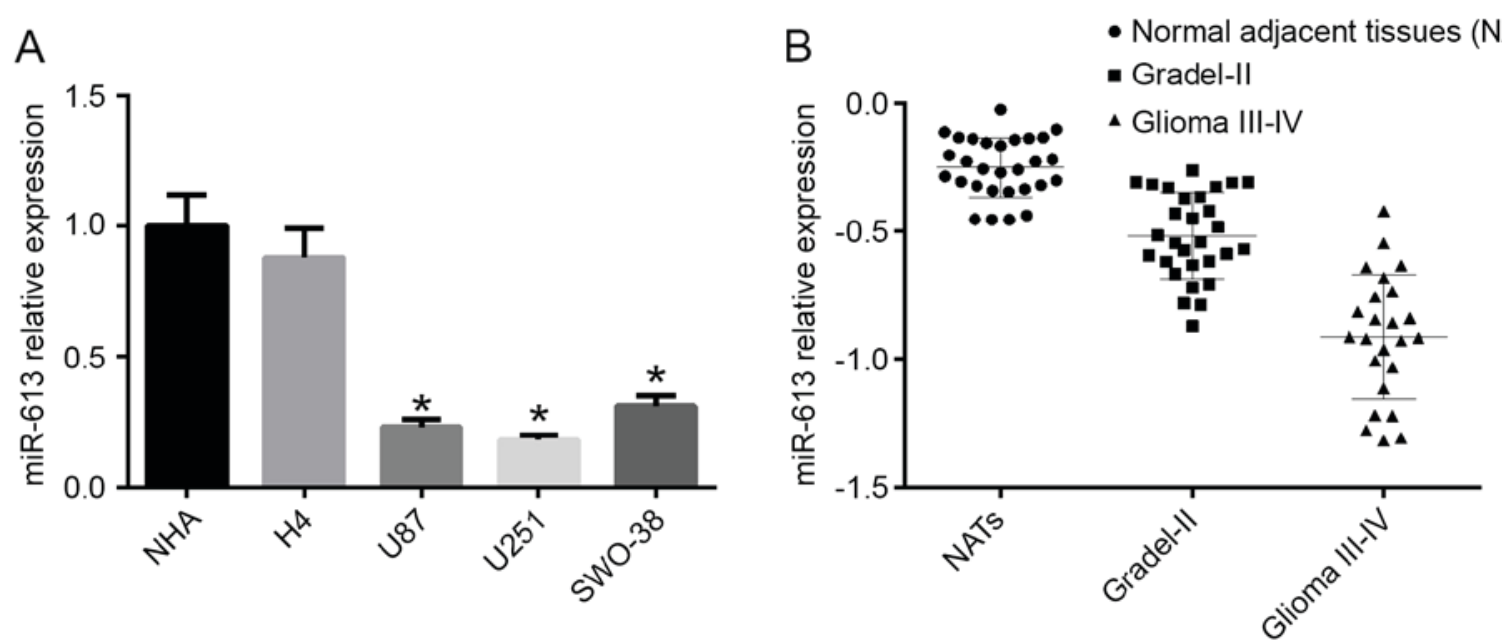

Figure 1. miR-613 is downregulated in glioma cells and high glioma grade. (A) The mRNA expression level of miR-613 was decreased in glioblastoma multiforme cells (U87, U215 and SWO-38) compared with low grade glioma cell lines (H4 and NHA). (B) The mRNA expression level of miR-613 was analyzed by reverse transcription-quantitative polymerase chain reaction the tissues of in patients. Data are expressed as the mean \pm standard error. "P<0.05. miR, microRNA.

A

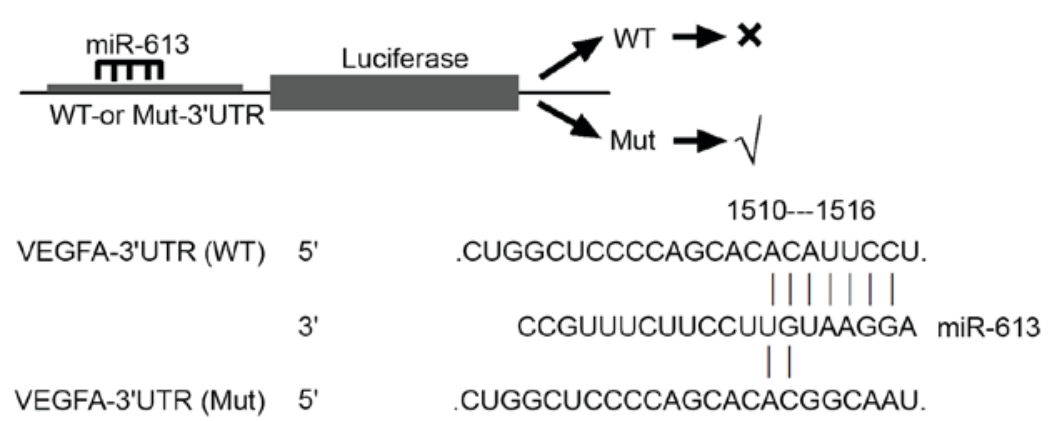

$\mathrm{B}$

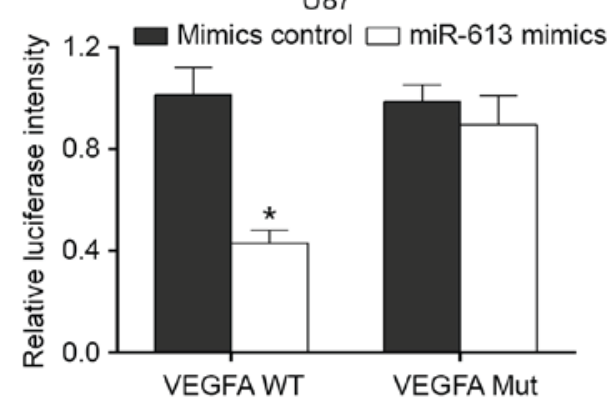

D

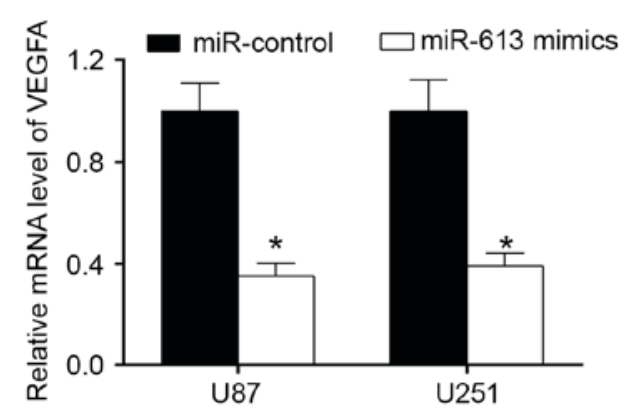

C

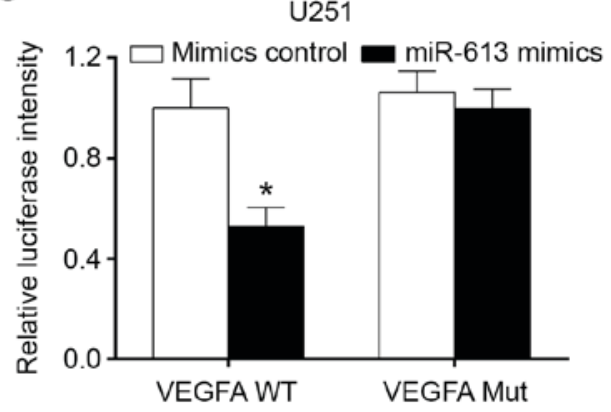

E

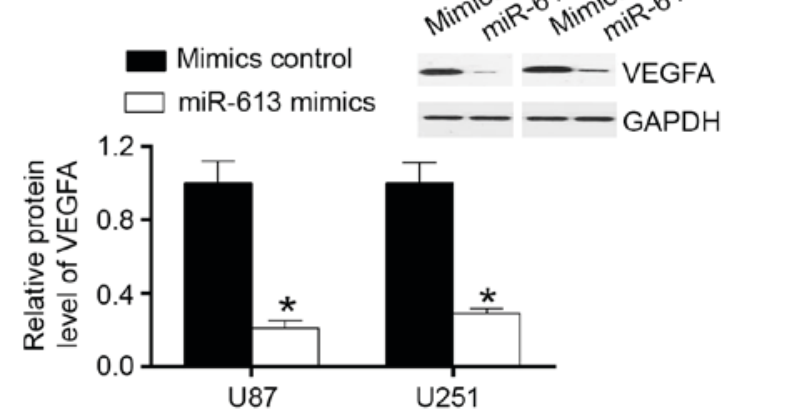

Figure 2. VEGFA is a direct target of miR-613. (A) Sequence alignment of miR-613 and the VEGFA 3'-UTR, which contains one predicted miR-613-biding site. Luciferase assay in (B) U87 and (C) U251 cell lines co-transfected with miR-613 and a luciferase reporter containing the VEGFA 3'-UTR WT or a Mut. (D) mRNA and (E) protein expression levels of VEGFA following miR-613 transfection. Data are expressed as the mean \pm standard error. ${ }^{*}<0.05$. miR, microRNA; WT, wild-type; Mut, mutant; VEGFA, vascular endothelial growth factor A; UTR, untranslated region. 
A

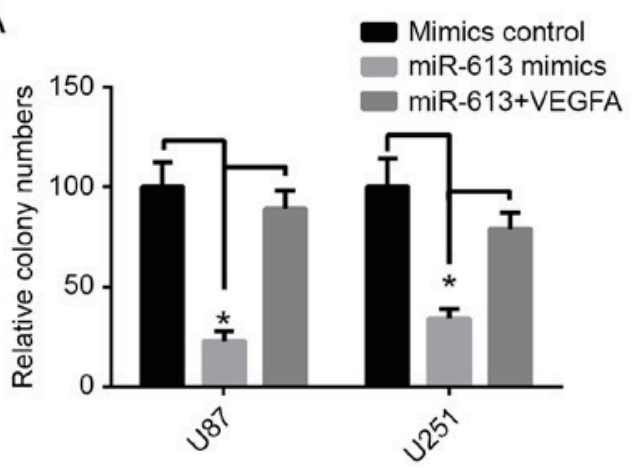

C

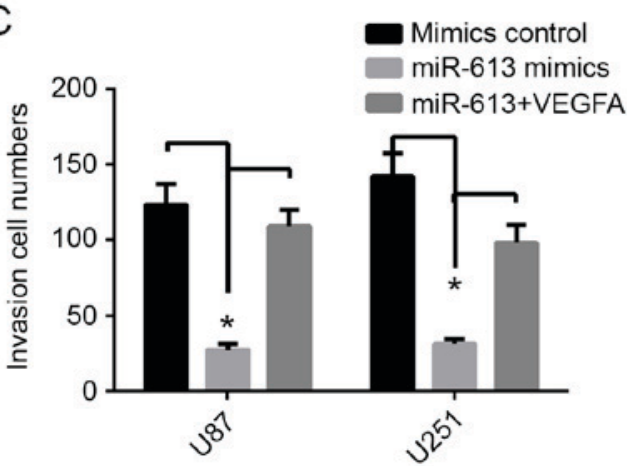

E

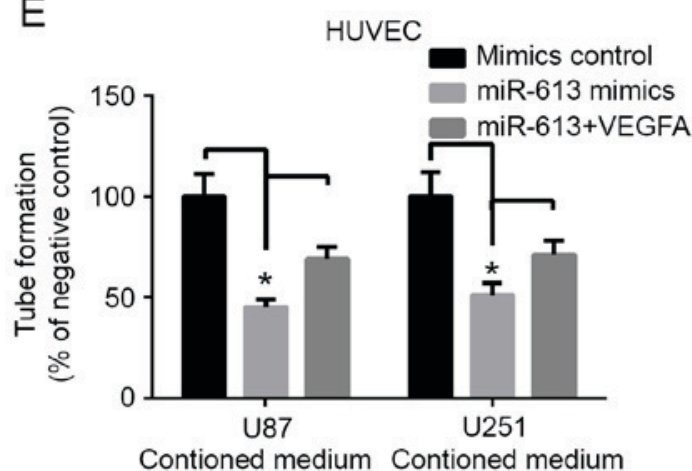

B

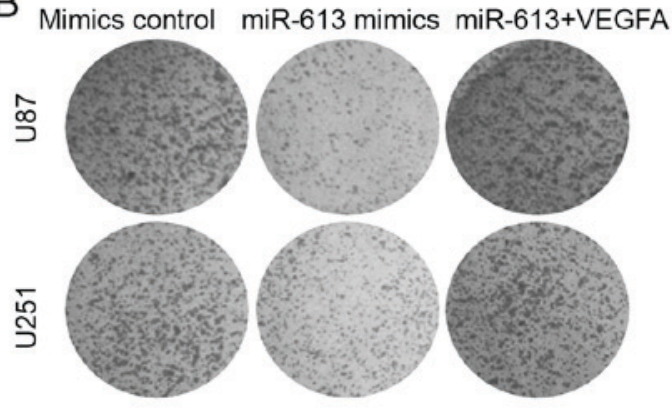

D

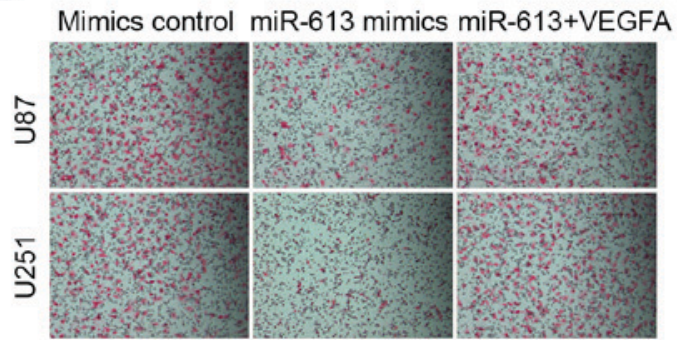

F

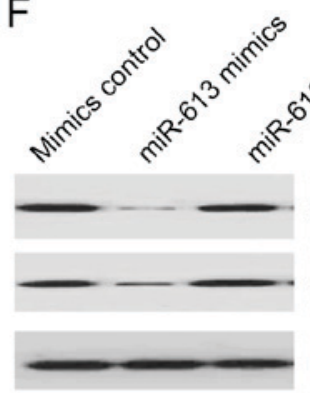

บ87
Contioned medium

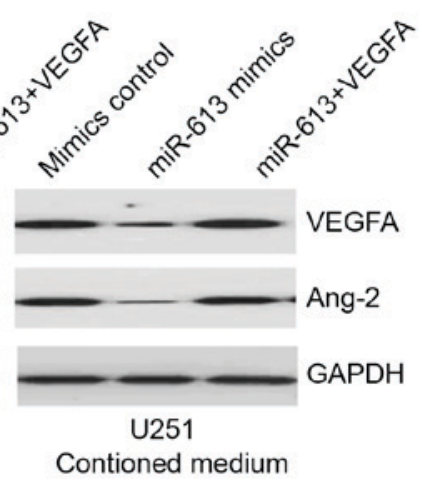

Figure 3. miR-613 suppresses cell proliferation and angiogenesis through targeting VEGFA. (A) Quantification of colony numbers of U251 and U87 cells following miR-163 or VEGFA transfection. (B) Representative images of colony formation assay using U251 and U87 cells transfected with control miRNA, miR-613 mimics, or miR-613 mimics and VEGFA. (C) Quantification of the invasive ability of U87 and U251 cells. (D) Representative images of invasion assay using U87 and U251 cells. Magnification, x100. (E) Tube formation of endothelial HUVEC cells after treatment with medium preconditioned by miR-613-overexpressing U87 and U251 cells or mock cells. (F) Representative western blot images of the protein expression levels of VEGFA and Ang-2 in U87 and U251 cells transfected with mimic control, miR-613 mimics or miR-613, and VEGFA. GAPDH served as a reference protein. Data are expressed as the mean \pm standard error. "P<0.05. miR, microRNA; VEGFA, vascular endothelial growth factor A; Ang-2, angiopoietin-2.

risk for glioma recurrence $(17,18)$. The expression level of miR-613 was decreased in GBM, and its inhibitory action on VEGFA was involved with miR-613, which may have a role in GBM carcinogenesis by downregulating the expression of VEGFA. Therefore, the effects of miR-613 overexpression and VEGFA restoration in GBM cells was investigated. In the colony formation assay, overexpression of miR-613 markedly decreased colony formation in both U87 and U251 cell lines (Fig. 3A and B). The invasion experiment results demonstrated that upregulation of miR-613 significantly inhibited invasion in both GBM cell lines, compared with the mimic controls (Fig. 3C and D). The tube formation assay of endothelial HUVEC cells was suppressed by treatment with medium preconditioned by miR-613-overexpressing U87 and U251 cells (Fig. 3E). In addition, western blot analysis indicated that restoration of VEGFA ameliorated the miR-613-induced downregulation of VEGFA (Fig. 3F). The growth and invasion of tumor depends on tumor angiogenesis, there are various angiogenic factors regulating this process. Ang-2 is one of the most important proteins for this process (19). In this study, the results demonstrated that overexpression of miR-613 markedly decreased the level of Ang-2 compared with control, and it was markedly improved by transfection of VEGFA (Fig. 3F). However, the decrease in colony formation, invasion and tube formation produced by stable upregulation of miR-613 was restored by transfecting VEGFA into both U87 and U251 cell lines (Fig. 3A-E). These results indicated that miR-613 markedly inhibits invasion, proliferation and angiogenesis, at least partially, via targeting VEGFA.

Upregulation of miR-613 inhibits angiogenesis and growth of GBM cells in vivo. Next, the role of miR-613 on tumor 

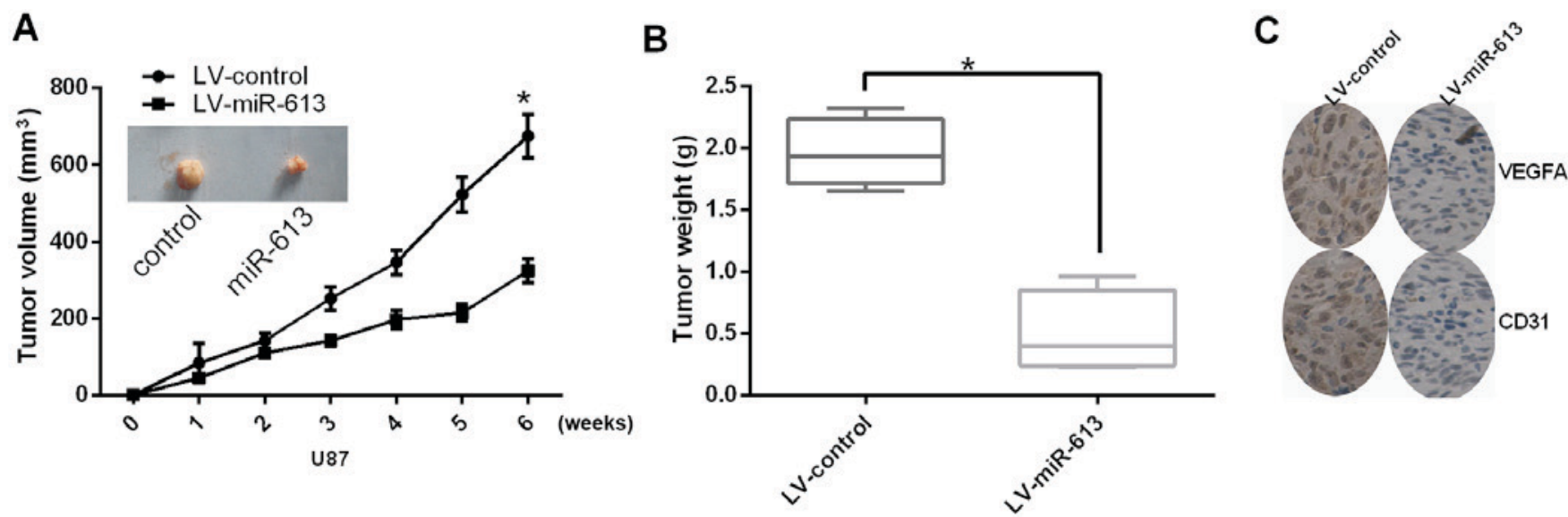

Figure 4. miR-613 weakens the growth and angiogenesis of glioblastoma multiforme cell line in vivo. (A) Stable transfection of U87 cells with miR-613 reduced the tumor size. (B) Overexpressing miR-613 significantly reduced the tumor weight. (C) Immuno-histochemical staining demonstrated that stable transfection of miR-613 caused the decrease of VEGFA and CD31 in vivo. Data are expressed as the mean \pm standard error. "P<0.05. miR, microRNA; VEGFA, vascular endothelial growth factor A; LV, lentivirus; CD31, cluster of differentiation 1.

angiogenesis and growth was studied in vivo. The tumor volume and tumor weight of subcutaneous xenograft tumors apparently decreased in nude mice with U87 cells transfected with miR-617, compared with those stably transfected with LV-control (Fig. 4A and B). Furthermore, the expression level of VEGFA was also reduced by stable transfection of miR-613. In addition, miR-613 decreased the protein expression levels of VEGFA and CD31 (Fig. 4C). In conclusion, these results indicated that miR-613 could suppress angiogenesis and growth of glioma cells in vivo.

\section{Discussion}

miRNAs serve key roles in many biological processes including proliferation, differentiation, development and apoptosis (20). Previous studies have demonstrated that miRNAs may represent potential therapeutic targets for a variety of diseases $(21,22)$. On the whole, there are two ways for developing miRNA-based therapeutics: miRNA mimics and miRNA antagonists. miRNA antagonists restrain endogenous miRNAs that exhibit gain-of-function in diseased tissues (23). miRNA mimics are used to recover loss-of-function. This approach, also termed 'miRNA replacement therapy', aims to transfect miRNAs into diseased cells that are regularly expressed in healthy cells (24). These miRNA-based treatment methods may be effective on tumor therapy. Although thousands of miRNAs have been detected in humans (25), only a few of them have been functionally characterized.

miR-613 was firstly reported to participate in lipid metabolism in macrophages (26) and HepG2 cells (27). Previous studies demonstrated a link between miR-613 and tumorigenesis $(11,28,29)$. The present study detected the expression level of miR-613 by RT-qPCR, and the results demonstrated that the mRNA expression level of miR-613 was decreased in high grade glioma cell lines (U87, U251 and SWO-38) compared with NHA cells and the H4 low-grade glioma cell line. Furthermore, the level of miR-613 was markedly associated with the grade of glioma, and the mRNA expression level of miR-613 was decreased in the poor development of glioma. In order to further investigate the potential therapeutic effect of overexpression of miR-613 in GBM cells, the role of miR-613 in the growth of subcutaneous xenograft tumors in nude mice was examined. Consistent with the result in vitro, restoration of miR-613 inhibited the growth of xenograft tumors. Studies also identified that upregulation of miR-613 reduced tumor angiogenesis and invasion in vitro (28,30-32). These results emphasized the therapeutic value of recovery of miR-613 expression level in the therapy of tumors.

Angiogenesis serves an important role in tumor growth and tumor progression. A large number of studies have demonstrated VEGFA as the most potential adjuster of tumor-induced angiogenesis in glioma $(15,16)$. Increased the expression of VEGFA is associated with aggravation of risk for glioma recurrence, poor prognosis and increased tumor microvessel density $(17,18)$. In the present study, miR-613-overexpressing cells were transfected with VEGFA, and examined the colony forming ability, the invasion ability and tube formation recovery. Certain direct targets of miR- 613 have been confirmed $(26,29)$. The present study identified a novel direct target of miR-613 and VEGFA. The results revealed that miR-613 had the ability to negatively modulate the expression of VEGFA, which serves important roles by connecting with a site in the VEGFA 3'-UTR. VEGFA increased the expression level of VEGFA in miR-613-overexpressing U87 and U251 cells. Ang-2 is another important protein to regulate tumor angiogenesis (19). The present study demonstrated that Ang-2 and VEGFA serve synergistic roles in tumor angiogenesis (33). Therefore, it was concluded that VEGFA may be a direct target for miR-613, implicating miR-613 as a novel target for glioma therapy.

In the present study, there were many lines of evidence to confirm the interaction between miR-613 and VEGFA. Firstly, the 3'-UTR of VEGFA had an assumed binding site of miR-613. Secondly, miR-613 inhibited the activity of a luciferase reporter merged with the 3'-UTR of VEGFA mRNA. Furthermore, miR-613 suppressed the protein and mRNA expression levels of VEGFA. These findings facilitate understanding of the role of VEGFA in regulating glioma cells.

In conclusion, the results of the present study suggested that miR-613 is involved in glioma. In addition, it was demonstrated 
that miR-613 serves an important role in the malignancy of glioma cells via inhibition of VEGFA expression. To the best of our knowledge, this study was the first to assess the association between miR-613 and VEGFA in glioma. The results implicate miR-613 as a potential target to treat gliomas in the future.

\section{Acknowledgements}

The present study was supported by the Natural Science Foundation of Shanxi, China (grant no. 2013011052-2).

\section{References}

1. Sun J, Zheng G, Gu Z and Guo Z: MiR-137 inhibits proliferation and angiogenesis of human glioblastoma cells by targeting EZH2. J Neurooncol 122: 481-489, 2015.

2. Zhang Y, Zhao S and Xu Z: Network and pathway analysis of microRNAs, transcription factors, target genes and host genes in human glioma. Oncol Lett 11: 3534-3542, 2016.

3. Lefranc F, Rynkowski M, DeWitte O and Kiss R: Present and potential future adjuvant issues in high-grade astrocytic glioma treatment. Adv Tech Stand Neurosurg 34: 3-35, 2009.

4. Stupp R, Hegi ME, Mason WP, van den Bent MJ, Taphoorn MJ, Janzer RC, Ludwin SK, Allgeier A, Fisher B, Belanger K, et al: Effects of radiotherapy with concomitant and adjuvant temozolomide versus radiotherapy alone on survival in glioblastoma in a randomised phase III study: 5-year analysis of the EORTC-NCIC trial. Lancet Oncol 10: 459-466, 2009.

5. Yue X, Wang P, Xu J, Zhu Y, Sun G, Pang Q and Tao R: MicroRNA-205 functions as a tumor suppressor in human glioblastoma cells by targeting VEGF-A. Oncol Rep 27: 1200-1206, 2012.

6. Krol J, Loedige I and Filipowicz W: The widespread regulation of microRNA biogenesis, function and decay. Nat Rev Genet 11: 597-610, 2010.

7. Dykxhoorn DM: MicroRNAs and metastasis: Little RNAs go a long way. Cancer Res 70: 6401-6406, 2010.

8. Di Leva G, Garofalo M and Croce CM: MicroRNAs in cancer. Annu Rev Pathol 9: 287-314, 2014.

9. Ciafrè SA, Galardi S, Mangiola A, Ferracin M, Liu CG, Sabatino G, Negrini M, Maira G, Croce CM and Farace MG: Extensive modulation of a set of microRNAs in primary glioblastoma. Biochem Biophys Res Commun 334: 1351-1358, 2005.

10. Moller HG, Rasmussen AP, Andersen HH, Johnsen KB, Henriksen M and Duroux M: A systematic review of microRNA in glioblastoma multiforme: Micro-modulators in the mesenchymal mode of migration and invasion. Mol Neurobiol 47: 131-144, 2013.

11. Fu X, Cui Y, Yang S, Xu Y and Zhang Z: MicroRNA-613 inhibited ovarian cancer cell proliferation and invasion by regulating KRAS. Tumour Biol 37: 6477-6483, 2016.

12. Zhao Q, Li J, Yan J, Liu S, Guo Y, Chen D and Luo Q: Lycium barbarum polysaccharides ameliorates renal injury and inflammatory reaction in alloxan-induced diabetic nephropathy rabbits. Life Sci 157: 82-90, 2016

13. Livak KJ and Schmittgen TD: Analysis of relative gene expression data using real-time quantitative PCR and the 2(-Delta Delta C(T)) method. Methods 25: 402-408, 2001.

14. Zhang H, Qi M, Li S, Qi T, Mei H, Huang K, Zheng L and Tong Q: microRNA-9 targets matrix metalloproteinase 14 to inhibit invasion, metastasis, and angiogenesis of neuroblastoma cells. Mol Cancer Ther 11: 1454-1466, 2012.

15. Kim KJ, Li B, Winer J, Armanini M, Gillett N, Phillips HS and Ferrara N: Inhibition of vascular endothelial growth factor-induced angiogenesis suppresses tumour growth in vivo. Nature 362: 841-844, 1993.
16. Plate KH, Breier G, Weich HA and Risau W: Vascular endothelial growth factor is a potential tumour angiogenesis factor in human gliomas in vivo. Nature 359: 845-848, 1992.

17. Chaudhry IH, O'Donovan DG, Brenchley PE, Reid H and Roberts IS: Vascular endothelial growth factor expression correlates with tumour grade and vascularity in gliomas. Histopathology 39: 409-415, 2001.

18. Varlet P, Guillamo JS, Nataf F, Koziak M, Beuvon F and DaumasDuport C: Vascular endothelial growth factor expression in oligodendrogliomas: A correlative study with Sainte-Anne malignancy grade, growth fraction and patient survival. Neuropathol Appl Neurobiol 26: 379-389, 2000.

19. Maisonpierre PC, Suri C, Jones PF, Bartunkova S, Wiegand SJ, Radziejewski C, Compton D, McClain J, Aldrich TH, Papadopoulos N, et al: Angiopoietin-2, a natural antagonist for Tie2 that disrupts in vivo angiogenesis. Science 277: 55-60, 1997.

20. Wang D, Qiu C, Zhang H, Wang J, Cui Q and Yin Y: Human microRNA oncogenes and tumor suppressors show significantly different biological patterns: From functions to targets. PLoS One 5: pii: e13067, 2010.

21. Volinia S, Calin GA, Liu CG, Ambs S, Cimmino A, Petrocca F, Visone R, Iorio M, Roldo C, Ferracin M, et al: A microRNA expression signature of human solid tumors defines cancer gene targets. Proc Natl Acad Sci USA 103: 2257-2261, 2006.

22. Lee YS and Dutta A: MicroRNAs in cancer. Annu Rev Pathol 4: 199-227, 2009.

23. Kota J, Chivukula RR, O'Donnell KA, Wentzel EA, Montgomery CL, Hwang HW, Chang TC, Vivekanandan P, Torbenson M, Clark KR, et al: Therapeutic microRNA delivery suppresses tumorigenesis in a murine liver cancer model. Cell 137: 1005-1017, 2009.

24. Bader AG, Brown D and Winkler M: The promise of microRNA replacement therapy. Cancer Res 70: 7027-7030, 2010.

25. Zhao XM, Liu KQ, Zhu G, He F, Duval B, Richer JM, Huang DS, Jiang CJ, Hao JK and Chen L: Identifying cancer-related microRNAs based on gene expression data. Bioinformatics 31: 1226-1234, 2015

26. Zhao R, Feng J and He G: miR-613 regulates cholesterol efflux by targeting LXRa and ABCA1 in PPAR $\gamma$ activated THP-1 macrophages. Biochem Biophys Res Commun 448: 329-334, 2014.

27. Zhong D, Zhang Y, Zeng YJ, Gao M, Wu GZ, Hu CJ, Huang G and He FT: MicroRNA-613 represses lipogenesis in HepG2 cells by downregulating LXRa. Lipids Health Dis 12: 32, 2013.

28. Ren W, Li C, Duan W, Du S, Yang F, Zhou J and Xing J: MicroRNA-613 represses prostate cancer cell proliferation and invasion through targeting Frizzled7. Biochem Biophys Res Commun 469: 633-638, 2016.

29. Li D, Li DQ, Liu D and Tang XJ: MiR-613 induces cell cycle arrest by targeting CDK4 in non-small cell lung cancer. Cell Oncol (Dordr) 39: 139-147, 2016.

30. Li B, Xie Z, Li Z, Chen S and Li B: MicroRNA-613 targets FMNL2 and suppresses progression of colorectal cancer. Am J Transl Res 8: 5475-5484, 2016.

31. Qiu W, Yang Z, Fan Y and Zheng Q: MicroRNA-613 inhibits cell growth, migration and invasion of papillary thyroid carcinoma by regulating SphK2. Oncotarget 7: 39907-39915, 2016.

32. Yu H, Duan P, Zhu H and Rao D: miR-613 inhibits bladder cancer proliferation and migration through targeting SphK1. Am J Transl Res 9: 1213-1221, 2017.

33. Scheuer W, Thomas M, Hanke P, Sam J, Osl F, Weininger D, Baehner M, Seeber S, Kettenberger H, Schanzer J, et al: Anti-tumoral, anti-angiogenic and anti-metastatic efficacy of a tetravalent bispecific antibody (TAvi6) targeting VEGF-A and angiopoietin-2. MAbs 8: 562-573, 2016. 